\title{
Generalized synchronization between chimera states
}

\author{
Ralph G. Andrzejak, ${ }^{1,2}$ Giulia Ruzzene, ${ }^{1}$ and Irene Malvestio ${ }^{1,3,4}$ \\ ${ }^{1}$ Department of Information and Communication Technologies, Universitat Pompeu Fabra, \\ Barcelona, Catalonia, Spain \\ ${ }^{2}$ Institute for Bioengineering of Catalonia (IBEC), Barcelona, Catalonia, Spain \\ ${ }^{3}$ Department of Physics and Astronomy, University of Florence, Sesto Fiorentino, Italy \\ ${ }^{4}$ Institute for Complex Systems, CNR, Sesto Fiorentino, Italy
}

(Received 12 April 2017; accepted 9 May 2017; published online 23 May 2017)

\begin{abstract}
Networks of coupled oscillators in chimera states are characterized by an intriguing interplay of synchronous and asynchronous motion. While chimera states were initially discovered in mathematical model systems, there is growing experimental and conceptual evidence that they manifest themselves also in natural and man-made networks. In real-world systems, however, synchronization and desynchronization are not only important within individual networks but also across different interacting networks. It is therefore essential to investigate if chimera states can be synchronized across networks. To address this open problem, we use the classical setting of ring networks of non-locally coupled identical phase oscillators. We apply diffusive drive-response couplings between pairs of such networks that individually show chimera states when there is no coupling between them. The drive and response networks are either identical or they differ by a variable mismatch in their phase lag parameters. In both cases, already for weak couplings, the coherent domain of the response network aligns its position to the one of the driver networks. For identical networks, a sufficiently strong coupling leads to identical synchronization between the drive and response. For non-identical networks, we use the auxiliary system approach to demonstrate that generalized synchronization is established instead. In this case, the response network continues to show a chimera dynamics which however remains distinct from the one of the driver. Hence, segregated synchronized and desynchronized domains in individual networks congregate in generalized synchronization across networks. Published by AIP Publishing.
\end{abstract}

[http://dx.doi.org/10.1063/1.4983841]

Notwithstanding their simple structure, networks of coupled oscillators can show intriguingly complex dynamics. In a classical setting, ${ }^{1}$ identical phase oscillators are arranged on a ring and are connected by a nonlocal coupling, which has the same form for all oscillators. Despite this translational symmetry of the network, the oscillators can spontaneously form two complementary groups. While a group of oscillators rotates coherently, the remaining oscillators perform an erratic motion. This surprising co-existence of synchronous and asynchronous motion in a system of identical oscillators was named chimera state $^{2}$ and was subsequently found for a rich variety of different network topologies, oscillator types, and coupling schemes. ${ }^{3,4}$ So far, most work has focussed on chimera states in isolated networks. Realworld networks, however, are typically not isolated but connected to other networks. It is therefore essential to investigate the interplay of chimera states across separate networks. We here demonstrate that a simple coupling of oscillators across networks allows one to induce different types of synchronization between the networks. In particular, this includes generalized synchronization, where the state of a driving network fully determines the state of a response network, while both networks still show chimera states with distinct spatiotemporal dynamics. Hence, our results show that the co-existence of synchronous and asynchronous motion, which is the essence of chimera states, is not limited to individual networks but can spread across interacting networks. Our work can therefore lead to a broader applicability of the concept of chimera states to real-world phenomena.

\section{INTRODUCTION}

In the past few years, many reports on the experimental observation of chimera states were published..$^{5-16}$ Furthermore, an increasing number of conceptual links have been established between chimera states and a broad variety of natural and man-made dynamics. From early on this concerned neuronal dynamics (e.g., Ref. 17). Subsequently, links were drawn to unihemispheric sleep, ${ }^{18-20}$ information processing in biological networks, ${ }^{21}$ molecular cell biology dynamics, ${ }^{22}$ epileptic seizures, ${ }^{23,24}$ social dynamics of cross cultural interactions, ${ }^{25}$ dynamics of ecosystems,${ }^{26}$ evolutionary dynamics in biological, social, and ecological systems, ${ }^{27,28}$ spin torque nano-oscillators, ${ }^{29}$ and quorum sensing mechanisms. ${ }^{30}$ Little is known however about the interplay of chimera states across separate networks. Recent studies showed that bidirectional couplings between networks can suppress $^{31,32}$ or induce ${ }^{30-32}$ chimera states in individual networks, and coupling delays were found to play an important role in this multiplexing setting. ${ }^{30,32}$ Co-existing chimeras were reported to be either identical or non-identical across networks. ${ }^{31}$ However, from these studies ${ }^{30-32}$ one cannot 
conclude if chimera states can be synchronized across networks. We aim to close this gap by demonstrating generalized synchronization between chimera states.

The discovery of generalized synchronization ${ }^{33}$ and phase synchronization $^{34}$ set milestones in the applicability of dynamical systems theory to real-world dynamics. It led beyond preceding studies ${ }^{35,36}$ by showing that synchronization between chaotic dynamics is not restricted to identical synchronization between identical dynamics. Instead synchronization can be attained between dynamics that differ in their parameters or even have different governing equations. A further major step extending the scope of these concepts was to proceed from pairs of dynamics ${ }^{33,34,36-38}$ to pairs of networks of dynamics. ${ }^{39-42}$ In drive-response network pairs, the node dynamics in each network are coupled to other nodes within their own network. Moreover, nodes in the driver network are coupled unidirectionally to nodes in the response network. Pioneering work showed that identical synchronization between all nodes in the driver network and their counterpart nodes in the response network can be attained between two identical networks ${ }^{39}$ and between networks that are identical except for the coupling topology within networks. ${ }^{39,40}$ The term outer synchronization was coined to distinguish such synchronization between networks from inner synchronization within networks. ${ }^{39}$ Subsequent work introduced further differences between the drive and response networks. Apart from differing network topologies, studies dealt with nodes that obeyed differing governing equations ${ }^{41,42}$ including dynamics of differing dimensionality ${ }^{41}$ Furthermore, networks comprised by unequal numbers of nodes for the drive and response were analyzed. ${ }^{42}$ While in these cases identical synchronization is impossible, control schemes of the drive-response coupling still allow achieving generalized synchronization. ${ }^{41,42}$

A priori it is not clear whether these findings carry over to networks that individually show chimera states when there is no coupling between them. Networks capable of showing chimera states are often multi-stable, and chimera states can switch between co-existing attractors. In finite-size networks chimera states are known to eventually collapse to a fully coherent state, ${ }^{43}$ and thus there might not be enough time to reach a synchronized motion prior to this collapse. Furthermore, across network coupling can suppress chimera states. ${ }^{31,32}$ The input from the driver network might therefore destabilize the chimera state in the response network, potentially making generalized synchronization impossible. This work shows that this does not have to be the case and that generalized synchronization can be established, with the response network continuing to exhibit chimera states. This interplay between synchronization and persistent desynchronization within networks along with synchronization across networks does not require any elaborated control scheme but can be achieved with a simple diffusive drive-response coupling.

\section{METHODS}

Consider that the $m_{x}$-dimensional dynamics $X$ drives the $m_{y}$-dimensional dynamics $Y$ via a unidirectional coupling of strength $\varepsilon$

$$
\begin{gathered}
\dot{x}(t)=F(x(t)), \\
\dot{y}(t)=G(y(t), x(t), \varepsilon) .
\end{gathered}
$$

Here, $x(t) \in R^{m_{x}}$ and $y(t) \in R^{m_{y}}$ are the dynamical variables in the state spaces of $X$ and $Y$. One says that generalized synchronization is established if a transformation $H: X$ $\rightarrow Y$ exists such that $y(t)=H(x(t)) .^{33,37,38}$ In general, according to Eq. (2), the state of the response dynamics $y(t)$ is determined by the initial conditions $x\left(t_{0}\right)$ and $y\left(t_{0}\right)$. If the coupling $\varepsilon$ induces generalized synchronization, the existence of $H$ implies that $y(t)$ is fully determined by the state of the driver dynamics $x(t)$ and thereby by the initial conditions $x\left(t_{0}\right)$ only. Accordingly, for generalized synchronization $y(t)$ is independent from its own initial conditions $y\left(t_{0}\right)$. This observation leads to a straightforward way to numerically test the generalized synchronization, the auxiliary system approach. ${ }^{37,38,42}$ One introduces a replica $y^{\prime}(t)$ of the response dynamics $y(t)$, the so-called auxiliary system $\dot{y}^{\prime}(t)=G\left(y^{\prime}(t), x(t), \varepsilon\right)$ and starts it with different initial conditions $y^{\prime}\left(t_{0}\right) \neq y\left(t_{0}\right)$. Once generalized synchronization is established between the drive and the two identical responses, both the $y^{\prime}(t)$ and $y(t)$ are uniquely determined by $x(t)$. Therefore they must coincide, i.e., $y^{\prime}(t)$ $=y(t)$. Generalized synchronization between the drive and response can therefore be detected by identical synchronization between the two identical response dynamics. The detection of this identical synchronization is numerically straightforward, while the direct detection of the existence of $H$ would be much more involved.

We use the auxiliary system approach to detect generalized synchronization between two rings of each $N$ identical phase oscillators. In the framework sketched above this corresponds to $m_{x}=m_{y}=N$. Within each ring, the nodes are coupled via a rectangular kernel with broadness $2 b$. The phases $\phi_{j}(t)$ of the driving network $\Phi$ are governed by

$$
\dot{\phi}_{j}(t)=\omega-\frac{1}{2 b} \sum_{k=j-b}^{j+b} \sin \left(\phi_{j}(t)-\phi_{k}(t)+\alpha_{\mathrm{D}}\right) .
$$

Here, $\omega$ is the natural frequency, and $\alpha_{D}$ is the phase lag parameter of the driving network. Throughout this text, $j=1, \ldots, N$, and according to the ring architecture, sums and differences of oscillator indices are to be understood as modulo $N .{ }^{44}$ We use $N=50, b=18$, and $\alpha_{\mathrm{D}}=1.46$. We took this particular value of the phase lag parameter and the ratio $N / b \approx 0.35$ from previous work, ${ }^{43,45}$ thereby avoiding the risk of tuning these parameters with regard to our results. At these parameters $\Phi$ shows chimera states, it segregates into two distinct groups. In the high-coherence group all nodes are locked to a narrow range of phases and jointly oscillate at an almost constant phase velocity. All remaining nodes form the low-coherence group. For these oscillators the phases are dispersed and evolve irregularly. That means the spatial symmetry of the network structure is broken by its dynamics.

The response network $\Psi$ has the same core structure like the driving network $\Phi$. In addition to its connections within $\Psi$, however, each individual oscillator $j$ receives a 
unidirectional coupling with strength $\varepsilon$ from oscillator $j$ in $\Phi$. The phases of $\Psi$ are thus determined by

$$
\begin{aligned}
\dot{\psi}_{j}(t)= & \omega-\varepsilon \sin \left(\psi_{j}(t)-\phi_{j}(t)\right) \\
& -\frac{1}{2 b} \sum_{k=j-b}^{j+b} \sin \left(\psi_{j}(t)-\psi_{k}(t)+\alpha_{\mathrm{R}}\right) .
\end{aligned}
$$

The natural frequency is the same for both networks and can be set to $\omega=0$. The symmetry between the driving and response networks is only broken by using $\alpha_{R}=\alpha_{D}+\Delta \alpha$. The auxiliary response network $\Psi^{\prime}$ is a replica of $\Psi$. It is obtained from a second realization of Eq. (4), starting from different initial conditions but driven by the same $\phi_{j}(t)$. The inter-network coupling between individual oscillators induces a correspondence between oscillators with the same indices across the networks. We quantify the instantaneous differences between these oscillators by

$$
\delta_{\phi_{j} \psi_{j}}(t)=\left|\sin \left(\frac{\psi_{j}(t)-\phi_{j}(t)}{2}\right)\right|,
$$

where $|\cdot|$ denotes the absolute value. ${ }^{46}$ One can show that the expected value of Eq. (5) for phase differences drawn at random from $(0,2 \pi)$ is $2 / \pi$. The instantaneous difference between the overall network dynamics is naturally defined from the average difference between corresponding oscillators

$$
\delta_{\phi \psi}(t)=\frac{1}{N} \sum_{j=1}^{N} \delta_{\phi_{j} \psi_{j}}(t)
$$

The quantities $\delta_{\phi_{j} \psi_{j}^{\prime}}(t), \delta_{\phi \psi^{\prime}}(t), \delta_{\psi_{j} \psi_{j}^{\prime}}(t), \delta_{\psi \psi^{\prime}}(t)$ are defined analogously. Generalized synchronization between the drive $\Phi$ and response $\Psi$ can be detected from $\delta_{\psi \psi^{\prime}}(t)=0$, and identical synchronization is reflected in $\delta_{\phi \psi}(t)=\delta_{\phi \psi^{\prime}}(t)=0$.

Recall that the two response networks differ from the driver network only in their phase lag parameter via $\alpha_{R}$ $=\alpha_{\mathrm{D}}+\Delta \alpha$. We show results for identical $(\Delta \alpha=0)$, almost identical networks $(\Delta \alpha \rightarrow 0)$ and substantially different networks $(0.01 \leq \Delta \alpha \leq 0.08)$. Given their parameters, the uncoupled response systems show no clear chimera states for $\Delta \alpha>0.08$. For almost identical networks we used $\Delta \alpha$ $=10^{-\gamma}$, with $\gamma=3,4, \ldots, 15$. Even smaller differences cannot be studied. This is because in the common IEEE standard 754 for double precision, ${ }^{47} \alpha_{D}+\Delta \alpha$ becomes numerically indistinguishable from $\alpha_{\mathrm{D}}$ for $\Delta \alpha \leq 10^{-16}$. For each $\Delta \alpha$, we varied the coupling strength within $0 \leq \varepsilon \leq 1$.

We generated ten independent realizations of the dynamics for each combination of $\Delta \alpha$ and $\varepsilon$. For each realization, we started the networks $\Phi, \Psi$, and $\Psi^{\prime}$ with random initial phases uniformly distributed in $(0,2 \pi)$. We integrated the dynamics using a fourth-order Runge-Kutta scheme with a fixed sampling time of $d t=0.01$ for a total of $5 \times 10^{6}$ sampling times, corresponding to $5 \times 10^{4}$ dimensionless time units. During the first $10^{4}$ time units, we kept the coupling turned off $(\varepsilon=0)$. The transient from random initial phases to the formation of a chimera state in uncoupled networks always took less than $10^{3}$ time units. Accordingly, during the first $10^{4}$ time units, all three networks could settle to a chimera state not being influenced by any other dynamics. At $10^{4}$ time units the coupling was turned on for both $\Phi \rightarrow \Psi$ and $\Phi \rightarrow \Psi^{\prime}$. The time period of $4 \times 10^{4}$ time units between the onset of the coupling and the end of the simulation was found to be sufficiently long to test reliably whether or not the networks settled to a synchronized motion.

For our finite-size ring of phase oscillators, chimera states can suddenly collapse to a fully coherent state $^{43}$ in which the phases of all nodes become locked and oscillate at a constant phase velocity. Furthermore, after the initialization with random phases, the network can directly go to this fully coherent state without ever forming a chimera state. While chimera state collapses were studied in Refs. 24 and 43 , we exclude them from the present study. Whenever either of the networks went to the fully coherent state, the realization was discarded and a new realization was started with new random initial conditions. This had to be done only occasionally, since at the parameters we use the mean lifetime of chimera states is on the order of $10^{6}$ dimensionless time units. ${ }^{24,43}$ Accordingly, this lifetime is much higher than the time span of our simulation of $5 \times 10^{4}$ dimensionless time units.

\section{RESULTS}

We start by inspecting the network dynamics with the coupling turned off $\varepsilon=0$ and $\Delta \alpha=0.05$ [Figs. 1(a)-1(c)]. All networks are settled to a chimera state. The spatiotemporal phase pattern [Fig. 1(a)] reveals the division of the networks into their high- and low-coherence groups. The high-coherence groups can be recognized by vertical stripes, reflecting the joint oscillation of their nodes. In contrast, the low-coherence groups show an irregular spatio-temporal phase dynamics. Since the networks are uncoupled, there is no correspondence between oscillators across networks and their instantaneous differences $\delta_{\phi_{j} \psi_{j}}(t), \delta_{\phi_{j} \psi_{j}^{\prime}}(t)$, and $\delta_{\psi_{j} \psi_{j}^{\prime}}(t)$ are random [Fig. 1(b)]. Accordingly, $\delta_{\phi \psi}(t)$ and $\delta_{\psi \psi^{\prime}}(t)$ fluctuate around their expected value for random phase differences $2 / \pi$ [Fig. 1(c)]. The quantity $\delta_{\phi \psi^{\prime}}(t)$ behaves analogously to $\delta_{\phi \psi}(t)$ and is not shown to allow for a better distinction between $\delta_{\phi \psi}(t)$ and $\delta_{\psi \nu^{\prime}}(t)$. The slow variation in $\delta_{\psi \psi^{\prime}}(t)$ is caused by the drifting of the two complementary groups ${ }^{45}$ in both networks resulting in the transient overlap of their high coherence groups. A faster variation is found for $\delta_{\phi \psi}(t)$ since the networks' mean fields get alternately in-phase and out-of-phase due to their distinct mean frequencies. Both variations are also reflected in patterns in Fig. 1(b).

We now use a coupling strength $\varepsilon=0.2$ and keep $\Delta \alpha=0.05$ [Figs. 1(d)-1(f)]. First we note that the positions of the high-coherence groups of the response networks $\Psi$ and $\Psi^{\prime}$ align to the one in the driver network $\Phi$ [Fig. 1(d)]. As a consequence, the spatio-temporal plots of these differences themselves resemble chimera states, in the sense of being segregated in a regular and irregular region. While $\delta_{\phi_{j} \psi_{j}}(t), \delta_{\phi_{j} \psi_{j}^{\prime}}(t)$, and $\delta_{\psi_{j} \psi_{j}^{\prime}}(t)$ still cover their full range from 0 to 1 [Fig. 1(e)], the averaged measures $\delta_{\phi \psi}(t)$ and, in 

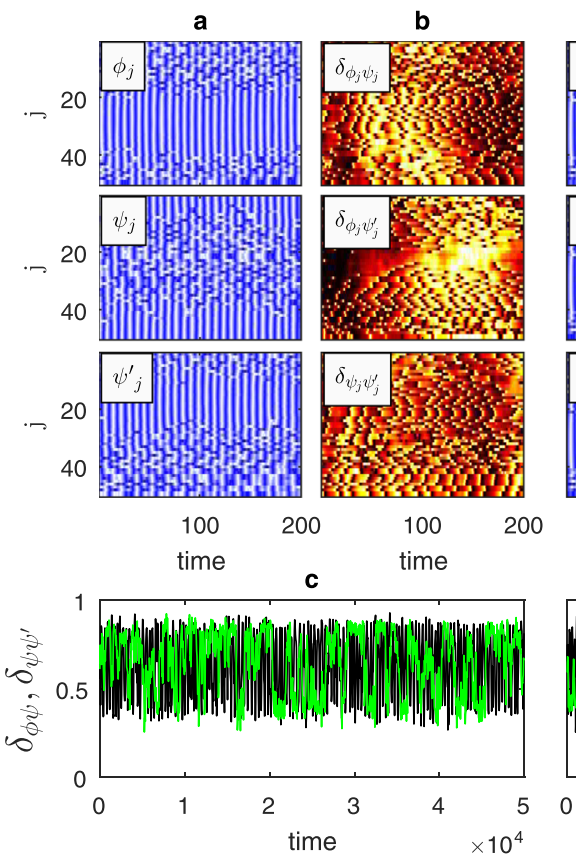
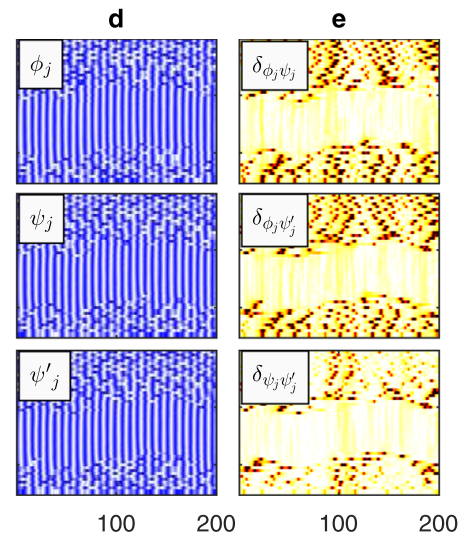

time

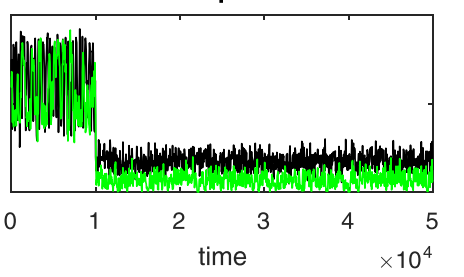

g
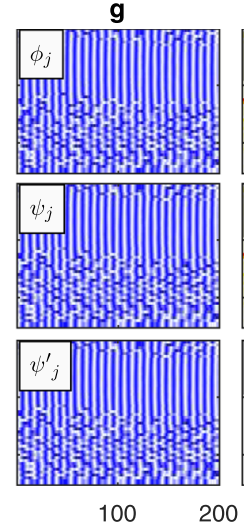

time
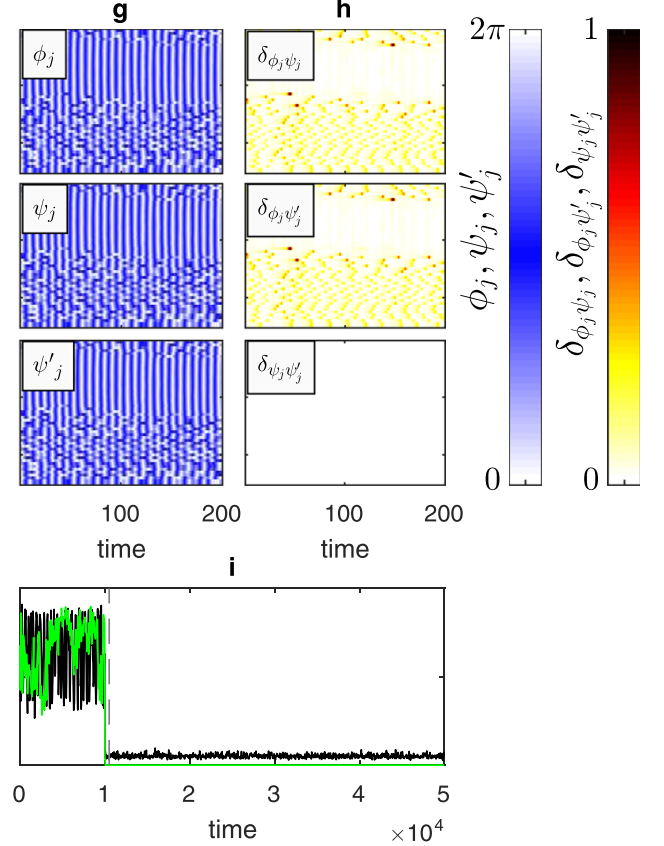

FIG. 1. For zero coupling $[\varepsilon=0$ (a)-(c) $]$ and weak coupling $[\varepsilon=0.2$ (d)-(f)] all three networks remain unsynchronized. For strong coupling $[\varepsilon=0.35$ (g)-(i) $]$ the two response networks show identical synchronization, reflecting generalized synchronization between the driver and response networks. Temporal evolution of phases (a), (d), and (g) and instantaneous differences between individual oscillators (b), (e), and (h) for the last 200 dimensionless time units prior to the end of the simulation. The instantaneous differences between overall networks dynamics (c), (f), and (i) are shown for the entire duration of the simulation [black: $\delta_{\phi \psi}(t)$; green: $\delta_{\psi \psi^{\prime}}(t)$ ]. The coupling was turned on at 10000 dimensionless time units. In panel (i) the vertical dashed line marks the time when $\delta_{\psi} \psi^{\prime}(t)$ becomes numerically indistinguishable from zero.

particular, $\delta_{\psi \psi^{\prime}}(t)$ drops to lower values directly after the onset of the coupling [Fig. 1(f)]. However, none of them reaches zero throughout the simulation. Hence, there is still no synchronization between either pair of the networks.

This changes for a higher coupling strength $\varepsilon=0.35$, with $\Delta \alpha=0.05$ kept fixed [Figs. 1(g)-1(i)]. The differences $\delta_{\phi_{j} \psi_{j}}(t)$ and $\delta_{\phi_{j} \psi_{j}^{\prime}}(t)$ are lower on average but remain nonzero. In contrast, $\delta_{\psi_{j} \psi_{j}^{\prime}}(t)$ is zero across time and all oscillators [Fig. 1(h)], and therefore $\delta_{\phi_{j} \psi_{j}}(t)$ and $\delta_{\phi_{j} \psi_{j}^{\prime}}(t)$ are identical. The profile of $\delta_{\psi \psi} \psi^{\prime}(t)$ shows that the drop to zero takes place shortly after the coupling is turned on [Fig. 1(i)]. In contrast, $\delta_{\phi \psi}(t)$ again decreases with regard to the level prior to the coupling, but it never takes zero values. This means that the driven networks $\Psi$ and $\Psi^{\prime}$ are synchronized identically, but both remain different from the driver network $\Phi$. The coupling received from $\Phi$ causes both $\Psi$ and $\Psi^{\prime}$ to forget their mutually different initial conditions; their state is fully determined by the state of $\Phi$. Hence, there exists a function $H$ such that $\Psi=\Psi^{\prime}=H(\Phi)$, and this function $H$ is not the identity. Accordingly, there is generalized synchronization, but not identical synchronization, between the driver network and the response networks. Both $\Psi$ and $\Psi^{\prime}$ continue to exhibit a chimera state despite being synchronized to $\Phi$. Since $H$ is not the identity function, their chimera state is not simply a replica of the one of $\Phi$. Importantly, the time it takes the response network to synchronize to the driver network is short compared to the duration of our simulation [Fig. 1(i)] and thereby even shorter as compared to the mean lifetime of the chimera states of our network.

We proceed by calculating the temporal averages of $\delta_{\phi \psi^{\prime}}(t)$ and $\delta_{\psi \psi^{\prime}}(t)$ across the last 20000 sampling times prior to the end of the simulation. We denote them by $\left\langle\delta_{\phi \psi^{\prime}}(t)\right\rangle$ and $\left\langle\delta_{\psi \psi} \psi^{\prime}(t)\right\rangle$ and show their dependence on $\varepsilon$ and $\Delta \alpha$ in Fig. 2. For zero couplings we find $\left\langle\delta_{\phi \psi^{\prime}}(t)\right\rangle \approx\left\langle\delta_{\psi \psi^{\prime}}(t)\right\rangle \approx 2 / \pi$, i.e., the value expected for independent networks. For nonzero couplings, both $\left\langle\delta_{\phi \psi^{\prime}}(t)\right\rangle$ and $\left\langle\delta_{\psi \psi^{\prime}}(t)\right\rangle$ decrease with increasing $\varepsilon$. In both cases this decrease becomes faster and more pronounced for smaller $\Delta \alpha$ values. However, as long as the driver and response networks are nonidentical $(\Delta \alpha>0)$, only $\left\langle\delta_{\psi \psi} \psi^{\prime}(t)\right\rangle$ reaches zero at a sufficient coupling. That means again that the two driven networks $\Psi$ and $\Psi^{\prime}$ synchronize identically, but there is no identical synchronization between the drive $\Phi$ and the response $\Psi$. Hence, there is generalized synchronization between $\Phi$ and $\Psi$. Even if the systems are almost identical with $\Delta \alpha$ approaching the limit of the numerical precision, $\left\langle\delta_{\phi \psi^{\prime}}(t)\right\rangle$ remains nonzero. We did not find a single realization for which $\Delta \alpha>0$ but $\left\langle\delta_{\phi \psi^{\prime}}(t)\right\rangle=0$. Identical synchronization between $\Phi$ and $\Psi$ was obtained only for identical systems.

\section{DISCUSSION}

In closing, we recall that at couplings which do not lead to generalized synchronization, the high coherence group of the response network aligns to the one of the driver network. This by itself is worth noting and adds to recent work on the control of chimeras. This control concerns the positions ${ }^{21,48-50}$ or relative $\operatorname{size}^{20}$ of the complementary groups forming chimera states, the stabilization of chimera states or their generation from the fully synchronous state. ${ }^{24,48,49,51,52}$ In particular, Bick and Martens envisioned that feedback control used to align chimeras across networks could be applied in hypothetical chimera computers. ${ }^{21}$ In our case no feedback control is needed to achieve this alignment 


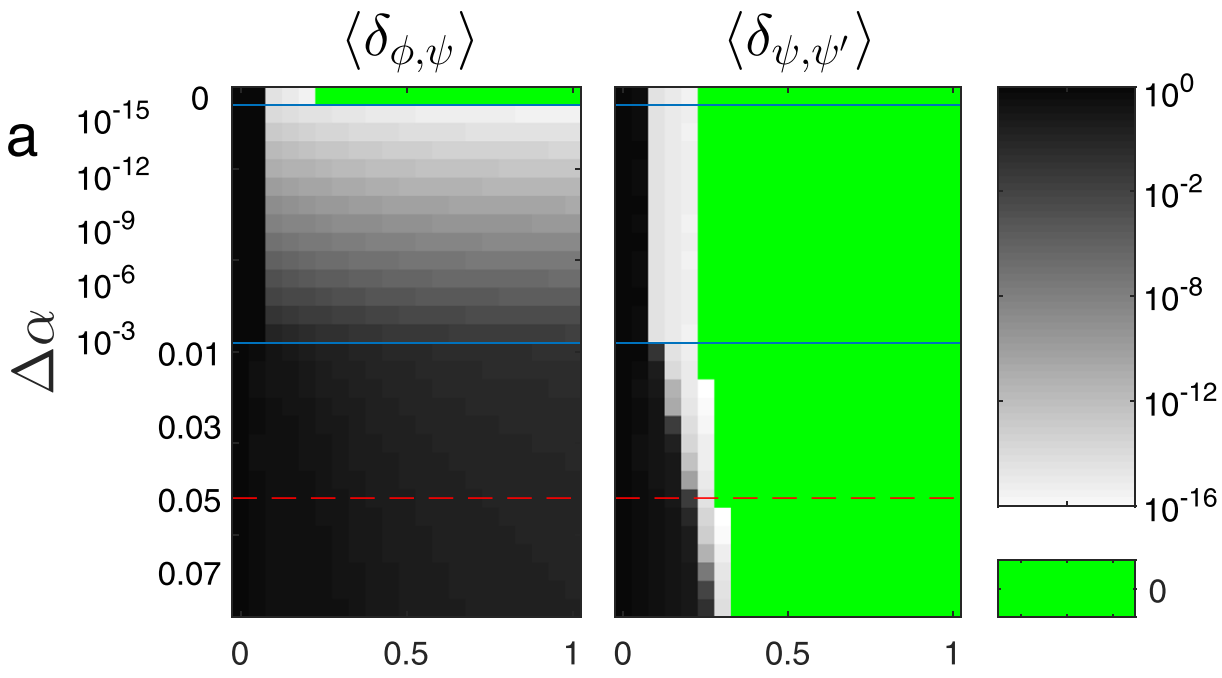

$\varepsilon$

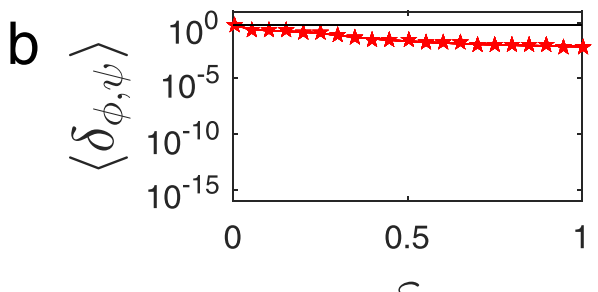

$\varepsilon$ $\varepsilon$

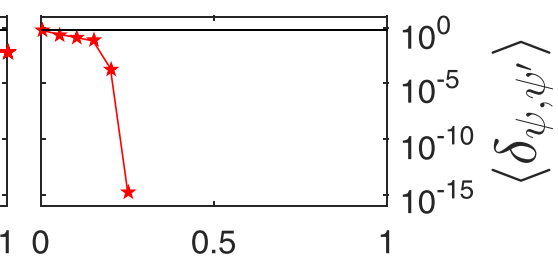

FIG. 2. (a) Dependence of the differences $\left\langle\delta_{\phi \psi}(t)\right\rangle$ and $\left\langle\delta_{\psi \psi^{\prime}}(t)\right\rangle$ on the coupling strength $\varepsilon$ and phase lag parameter detuning $\Delta \alpha$, averaged across ten independent realizations. Note the logarithmic color map scaling. Green is used when the differences were zero for all ten realizations. $\Delta \alpha$ is not varied uniformly but encompasses three settings separated by horizontal continuous blue lines: substantially different systems (bottom), almost identical systems (middle), and identical systems (top). An exemplary $\Delta \alpha$ used in panel (b) is marked by horizontal dashed red lines. (b) Dependence of $\left\langle\delta_{\phi \psi}(t)\right\rangle$ and $\left\langle\delta_{\psi \psi^{\prime}}(t)\right\rangle$ on the coupling strength $\varepsilon$ for an exemplary value of the phase lag parameter detuning $\Delta \alpha=0.05$, averaged across ten independent realizations. The vertical black lines mark the expected value of these differences for random phase differences $(2 / \pi)$. In the right panel, for $\varepsilon \geq 0.3$ we found $\left\langle\delta_{\psi \psi^{\prime}}(t)\right\rangle=0$ and therefore discontinued the curve in this logarithmic plot. The error bars depicting the ranges across realizations were too small to be discernible on the scales of the plots and are therefore not drawn. synchronization. Our main findings on generalized synchronization add an intriguing level to the chimera dynamics. We show that a network can maintain its inner segregation into synchronized and desynchronized domains and at the same time synchronize as a whole to another network, without simply replicating its dynamics. These theoretical findings may have a practical impact. Despite the ample experimental work, ${ }^{5-16}$ evidence for chimera states outside of such laboratory settings is still missing. ${ }^{3}$ Interacting networks are ubiquitous in nature, and synchronization is used to transmit information across networks. Therefore, while our setting is more complex than chimera states in individual networks, it might actually be the key in the search for real-world chimeras.

\section{ACKNOWLEDGMENTS}

We acknowledge funding from the Volkswagen foundation, the Spanish Ministry of Economy and Competitiveness, Grant No. FIS2014-54177-R, the CERCA Programme of the Generalitat de Catalunya (R.G.A. and G.R.), and from the European Union's Horizon 2020 research and innovation programme under the Marie Sklodowska-Curie Grant Agreement No. 642563 (R.G.A. and I.M.).

\footnotetext{
${ }^{1}$ Y. Kuramoto and D. Battogtokh, "Coexistence of coherence and incoherence in nonlocally coupled phase oscillators," Nonlinear Phenom. Complex Syst. 5, 380-385 (2002).

${ }^{2}$ D. M. Abrams and S. H. Strogatz, "Chimera states for coupled oscillators," Phys. Rev. Lett. 93, 174102 (2004).

${ }^{3}$ M. J. Panaggio and D. M. Abrams, "Chimera states: Coexistence of coherence and incoherence in networks of coupled oscillators," Nonlinearity $\mathbf{2 8}$, R67 (2015).
}

${ }^{4}$ D. M. Abrams, L. M. Pecora, and A. E. Motter, "Introduction to focus issue: Patterns of network synchronization," Chaos 26, 094601 (2016).

${ }^{5}$ A. M. Hagerstrom, T. E. Murphy, R. Roy, P. Hövel, I. Omelchenko, and E. Schöll, "Experimental observation of chimeras in coupled-map lattices," Nat. Phys. 8, 658-661 (2012).

${ }^{6}$ M. R. Tinsley, S. Nkomo, and K. Showalter, "Chimera and phase-cluster states in populations of coupled chemical oscillators," Nat. Phys. 8, 662-665 (2012).

${ }^{7}$ M. Wickramasinghe and I. Z. Kiss, "Spatially organized dynamical states in chemical oscillator networks: Synchronization, dynamical differentiation, and chimera patterns," PloS One 8, e80586 (2013).

${ }^{8}$ L. Larger, B. Penkovsky, and Y. Maistrenko, "Virtual chimera states for delayed-feedback systems," Phys. Rev. Lett. 111, 054103 (2013).

${ }^{9}$ E. A. Martens, S. Thutupalli, A. Fourrière, and O. Hallatschek, "Chimera states in mechanical oscillator networks," Proc. Natl. Acad. Sci. U. S. A. 110, 10563-10567 (2013).

${ }^{10}$ L. V. Gambuzza, A. Buscarino, S. Chessari, L. Fortuna, R. Meucci, and M. Frasca, "Experimental investigation of chimera states with quiescent and synchronous domains in coupled electronic oscillators," Phys. Rev. E 90, 032905 (2014).

${ }^{11}$ D. P. Rosin, D. Rontani, N. D. Haynes, E. Schöll, and D. J. Gauthier, "Transient scaling and resurgence of chimera states in networks of Boolean phase oscillators," Phys. Rev. E 90, 030902 (2014).

${ }^{12}$ L. Schmidt, K. Schönleber, K. Krischer, and V. García-Morales, "Coexistence of synchrony and incoherence in oscillatory media under nonlinear global coupling," Chaos 24, 013102 (2014).

${ }^{13}$ S. Nkomo, M. R. Tinsley, and K. Showalter, "Chimera and chimera-like states in populations of nonlocally coupled homogeneous and heterogeneous chemical oscillators," Chaos 26, 094826 (2016).

${ }^{14}$ K. Blaha, R. J. Burrus, J. L. Orozco-Mora, E. Ruiz-Beltrán, A. B. Siddique, V. D. Hatamipour, and F. Sorrentino, "Symmetry effects on naturally arising chimera states in mechanical oscillator networks," Chaos 26, 116307 (2016).

${ }^{15}$ J. D. Hart, K. Bansal, T. E. Murphy, and R. Roy, "Experimental observation of chimera and cluster states in a minimal globally coupled network," Chaos 26, 094801 (2016).

${ }^{16}$ J. Wojewoda, K. Czolczynski, Y. Maistrenko, and T. Kapitaniak, "The smallest chimera state for coupled pendula," Sci. Rep. 6, 34329 (2016).

${ }^{17} \mathrm{H}$. Sakaguchi, "Instability of synchronized motion in nonlocally coupled neural oscillators," Phys. Rev. E 73, 031907 (2006). 
${ }^{18}$ D. Abrams, R. Mirollo, S. Strogatz, and D. Wiley, "Solvable model for chimera states of coupled oscillators," Phys. Rev. Lett. 101, 084103 (2008).

${ }^{19}$ S. W. Haugland, L. Schmidt, and K. Krischer, "Self-organized alternating chimera states in oscillatory media," Sci. Rep. 5, 9883 (2015).

${ }^{20}$ N. Semenova, A. Zakharova, V. Anishchenko, and E. Schöll, "Coherenceresonance chimeras in a network of excitable elements," Phys. Rev. Lett. 117, 014102 (2016).

${ }^{21}$ C. Bick and E. A. Martens, "Controlling chimeras," New J. Phys. 17, 033030 (2015).

${ }^{22}$ D. R. Brumley, N. Bruot, J. Kotar, R. E. Goldstein, P. Cicuta, and M. Polin, "Long-range interactions, wobbles, and phase defects in chains of model cilia," Phys. Rev. Fluids 1, 081201 (2016).

${ }^{23} \mathrm{~A}$. Rothkegel and K. Lehnertz, "Irregular macroscopic dynamics due to chimera states in small-world networks of pulse-coupled oscillators," New J. Phys. 16, 055006 (2014).

${ }^{24}$ R. G. Andrzejak, C. Rummel, F. Mormann, and K. Schindler, "All together now: Analogies between chimera state collapses and epileptic seizures," Sci. Rep. 6, 23000 (2016).

${ }^{25}$ J. C. González-Avella, M. G. Cosenza, and M. San Miguel, "Localized coherence in two interacting populations of social agents," Physica A 399, 24-30 (2014).

${ }^{26} \mathrm{P}$. S. Dutta and T. Banerjee, "Spatial coexistence of synchronized oscillation and death: A chimeralike state,” Phys. Rev. E 92, 042919 (2015).

${ }^{27}$ J. Hizanidis, E. Panagakou, I. Omelchenko, E. Schöll, P. Hövel, and A. Provata, "Chimera states in population dynamics: Networks with fragmented and hierarchical connectivities," Phys. Rev. E 92, 012915 (2015).

${ }^{28}$ N. E. Kouvaris, R. J. Requejo, J. Hizanidis, and A. Diaz-Guilera, "Chimera states in a network-organized public goods game with destructive agents," Chaos 26, 123108 (2016).

${ }^{29}$ V. K. Chandrasekar, R. Gopal, A. Venkatesan, and M. Lakshmanan, "Mechanism for intensity-induced chimera states in globally coupled oscillators," Phys. Rev. E 90, 062913 (2014).

${ }^{30}$ S. Majhi, M. Perc, and D. Ghosh, "Chimera states in uncoupled neurons induced by a multilayer structure," Sci. Rep. 6, 39033 (2016).

${ }^{31}$ V. A. Maksimenko, V. V. Makarov, B. K. Bera, D. Ghosh, S. K. Dana, M. V. Goremyko, N. S. Frolov, A. A. Koronovskii, and A. E. Hramov, "Excitation and suppression of chimera states by multiplexing," Phys. Rev. E 94, 052205 (2016).

${ }^{32}$ S. Ghosh, A. Kumar, A. Zakharova, and S. Jalan, "Birth and death of chimera: Interplay of delay and multiplexing," EPL (Europhys. Lett.) 115, 60005 (2016).

${ }^{33}$ N. F. Rulkov, M. M. Sushchik, L. S. Tsimring, and H. D. I. Abarbanel, "Generalized synchronization of chaos in directionally coupled chaotic systems," Phys. Rev. E 51, 980-994 (1995).

${ }^{34}$ M. G. Rosenblum, A. S. Pikovsky, and J. Kurths, "Phase synchronization of chaotic oscillators," Phys. Rev. Lett. 76, 1804-1807 (1996).

${ }^{35} \mathrm{H}$. Fujisaka and T. Yamada, "Stability theory of synchronized motion in coupled-oscillator systems," Prog. Theor. Phys. 69, 32-47 (1983).
${ }^{36}$ L. M. Pecora and T. L. Carroll, "Synchronization in chaotic systems," Phys. Rev. Lett. 64, 821 (1990).

${ }^{37}$ H. D. I. Abarbanel, N. F. Rulkov, and M. M. Sushchik, "Generalized synchronization of chaos: The auxiliary system approach," Phys. Rev. E 53, 4528 (1996).

${ }^{38}$ L. Kocarev and U. Parlitz, "Generalized synchronization, predictability, and equivalence of unidirectionally coupled dynamical systems," Phys. Rev. Lett. 76, 1816 (1996).

${ }^{39} \mathrm{C}$. Li, W. Sun, and J. Kurths, "Synchronization between two coupled complex networks," Phys. Rev. E 76, 046204 (2007).

${ }^{40} \mathrm{H}$. Tang, L. Chen, J. Lu, and C. K. Tse, "Adaptive synchronization between two complex networks with nonidentical topological structures," Physica A 387, 5623-5630 (2008).

${ }^{41} \mathrm{X}$. Wu, W. X. Zheng, and J. Zhou, "Generalized outer synchronization between complex dynamical networks," Chaos 19, 013109 (2009).

${ }^{42} \mathrm{Y}$. Wu, C. Li, Y. Wu, and J. Kurths, "Generalized synchronization between two different complex networks," Commun. Nonlinear Sci. Numer. Simul. 17, 349-355 (2012).

${ }^{43} \mathrm{M}$. Wolfrum and O. E. Omel'chenko, "Chimera states are chaotic transients,” Phys. Rev. E 84, 015201 (2011).

${ }^{44}$ The Matlab source code for our simulations is available at the authors' web sites http://ntsa.upf.edu/.

${ }^{45}$ O. E. Omel'chenko, M. Wolfrum, and Y. L. Maistrenko, "Chimera states as chaotic spatiotemporal patterns," Phys. Rev. E 81, 065201 (2010).

${ }^{46} \mathrm{By} \mathrm{Eq}$. (5) phase differences ranging from 0 to $2 \pi$ are mapped to one sinus half wave, assigning the maximal difference of one to a phase difference of $\pi$. Without the division by two, phase differences of (close to) $\pi$ would count as (close to) zero. As an alternative one might use the order parameter of phase differences $\frac{1}{N}\left|\sum_{j} e^{i\left(\phi_{j}(t)-\psi_{j}(t)\right)}\right|$. However, due to its low absolute slope at small arguments, this order parameter is numerically indistinguishable from one for phase differences orders of magnitude higher than the limit of the numerical precision. In contrast, having an absolute slope of one at small arguments, Eq. (5) remains sensitive to phase differences down to this limit.

${ }^{47}$ IEEE Standards Committee, 754-2008, IEEE Standard for Floating-Point Arithmetic (IEEE Computer Society Standard, 2008), pp. 1-70.

${ }^{48}$ T. Isele, J. Hizanidis, A. Provata, and P. Hövel, "Controlling chimera states: The influence of excitable units," Phys. Rev. E 93, 022217 (2016).

${ }^{49}$ L. V. Gambuzza and M. Frasca, "Pinning control of chimera states," Phys. Rev. E 94, 022306 (2016).

${ }^{50}$ I. Omelchenko, O. E. Omel'chenko, A. Zakharova, M. Wolfrum, and E. Schöll, "Tweezers for chimeras in small networks," Phys. Rev. Lett. 116, 114101 (2016).

${ }^{51}$ J. Sieber, O. E. Omel'chenko, and M. Wolfrum, "Controlling unstable chaos: Stabilizing chimera states by feedback," Phys. Rev. Lett. 112, 054102 (2014).

${ }^{52}$ M. Wolfrum, O. E. Omel'chenko, and J. Sieber, "Regular and irregular patterns of self-localized excitation in arrays of coupled phase oscillators," Chaos 25, 053113 (2015). 